\title{
Homofobia internalizada e satisfação sexual em casais \\ homossexuais
}

\author{
Internalized Homophobia and sexual satisfaction on same-sex \\ couples
}

\section{Homofobia internalizada y satisfacción sexual en parejas homosexuales}

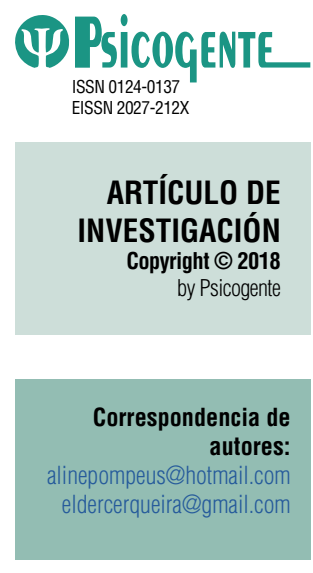

Recibido: $18-09-17$ Aceptado: 05-06-18 Publicado: 08-01-19

\author{
Aline Pompeu Silveira (iD) - Elder Cerqueira-Santos iD \\ Universidade Federal de Sergipe, São Cristóvão, Brasil
}

Resumo

Objetivo: O objetivo desta investigação foi observar a influência da religiosidade e da homofobia internalizada na satisfação sexual de casais do mesmo sexo.

Method: Um estudo foi conduzido com 94 participantes, 49 homens e 45 mulheres, vivendo em um relacionamento estável com parceiro do mesmo sexo. A primeira hipótese é que altos níveis de homofobia internalizada e religiosidade influenciam negativamente na satisfação sexual de participantes em relacionamentos sexuais do mesmo sexo. Os dados foram coletados em pessoa com um instrumento auto-aplicável abordando: dados sociodemográficos, homofobia internalizada, experiência de homofobia externa, satisfação sexual e índice de religiosidade. Comparações de médias intragrupos foram realizada com análises unidirecionais ANOVA entre as variáveis estudadas. Em seguida, foi realizado o teste de correlação de Pearson entre as variáveis e, finalmente, uma análise de regressão linear tendo a Satisfação Sexual como variável de desfecho.

Resultados: Os resultados indicaram níveis menores de satisfação sexual entre os grupos com maior homofobia internalizada, bem como uma diferença significante na satisfação de participantes com diferentes níveis de religiosidade, assim confirmando a hipótese prevista.

Conclusão: Se discute que homofobia internalizada prevê negativamente a satisfação sexual de um sujeito, já que interfere com seu bem estar e que outras variáveis, como abertura e religiosidade, também se mostram relevantes neste contexto.

Palavras-chave: Satisfação sexual; Homofobia Internalizada; Religiosidade

Abstract

Objective: This research aimed to observe the influence of the following variables: internalized homophobia and religiosity related to sexual satisfaction on people who develop a relationship with those of the same sex.

Method: 94 participants, 49 men and 45 women, living a stable relationship with a partner of the same sex, were sampled. The first hypothesis is that high levels of internalized homophobia and religiosity negatively impact on sexual satisfaction between people of same sex relationships. Data was collected by physical presence using a self-report questionaire focused on: socio-demographic, internalized homophobia, experience of external homophobia, sexual satisfaction and religiosity index. Comparisons with inter-groups mean through one-way analysis of variables ANOVA were considered. Then, Pearson's correlation test was performed between variables and, finally, an analysis of linear regression with sexual satisfaction as an outcome variable.

Results: Showed lower levels of sexual satisfaction among groups with the highest internalized homophobia, as well as a significant difference in relation to satisfaction of people at different levels of religiosity, confirming in this way, the predicted hypothesis.

Conclusion: It is discussed that internalized homophobia impacts negativetly on subject's sexual satisfaction as it interferes with their subjective well-being. Other variables, such as outness and religiosity, also show an impact on this context.

Key words: Sexual satisfaction; Internalized homophobia; Religiosity

Cómo citar este artículo (APA):

Pompeu Silveira, A. y Cerqueira-Santos, E. (2019). Homofobia internalizada y satisfacción sexual en parejas homosexuales. Psicogente 22(41), 1-18. https:// doi.org/10.17081/psico.22.41.3307 


\section{Resumen}

Objetivo: Observar la influencia de las variables homofobia internalizada y religiosidad en la satisfacción sexual de sujetos en relaciones con personas del mismo sexo.

Método: Se realizó un estudio con 94 sujetos, 49 hombres y 45 mujeres, viviendo en una relación estable con pareja del mismo sexo. La hipótesis inicial es que altos niveles de homofobia internalizada y de religiosidad influencian de forma negativa la satisfacción sexual de sujetos en relaciones LGB. Los datos fueron recolectados de forma presencial con cuestionario auto-aplicable abordando aspectos sociodemográficos, homofobia internalizada, homofobia externa sufrida, satisfacción sexual e índice de religiosidad. Se realizaron comparaciones de media intergrupos con análisis unidireccional ANOVA entre variables estudiadas. Se realizó la prueba de correlaciones de Pearson entre las variables y, por fin, un análisis de regresión lineal teniendo como resultado la variable satisfacción sexual.

Resultados: Estos apuntaron a menores niveles de satisfacción entre los grupos con mayor homofobia internalizada, así como para una diferencia significativa en la satisfacción de sujetos en niveles distintos de religiosidad, confirmando la hipótesis prevista.

Conclusiones: Se discute que la homofobia internalizada prevé negativamente la satisfacción sexual del sujeto, a medida que interfiere en su bienestar subjetivo, y que otras variables, como apertura y religiosidad, también tienen una influencia relevante en este contexto.

Palabras clave: Satisfacción sexual, Homofobia internalizada, Religiosidad

\section{INTRODUÇÃO}

A satisfação sexual é, dentre os fatores psicológicos, um dos mais estudados e avaliados no âmbito da sexualidade quando se trata de disfunções sexuais (Cardoso, 2003; Davis \& Petretic-Jackson, 2000; Pechorro, 2006; Souza, 2013). Segundo Mark, Garcia e Fisher (2015), nas últimas décadas, diversos estudo tem colocado a Satisfação Sexual como um fator importante para o bem-estar pessoal, mesmo em situações de não disfunção (Apt, Hurlbert, Pierce, \& White, 1996; Davison, Bell, LaChina, Holden, \& Davis, 2009).

É possível encontrar na literatura algumas definições para o conceito de Satisfação Sexual (Pechorro, Diniz, Almeida \& Vieira, 2009). Podendo ela estar associada a aspectos passados ou futuros da experiência sexual do indivíduo, assim como ser dividida em uma dimensão de satisfação sexual em geral ou com um parceiro específico (Davidson, Darling \& Norton, 1995; Delamater, 1991). Desta forma, a satisfação teria uma componente pessoal, que depende dos desejos da pessoa por determinados tipos e frequências de atividade sexual, e outra interpessoal, que depende dos tipos de comportamento do companheiro. Esta definição é similar àquela encontrada em Ashdown, Hackathorn e Clark (2011), em que satisfação sexual é definida como uma resposta afetiva advinda da avaliação, feita pelo indivíduo, de seu relacionamento sexual, incluindo a perceção de que as suas necessidades sexuais, bem como as expectativas do parceiro, estão sendo atendidas.

A partir destas definições, são construídos instrumentos e escalas que tem como intenção avaliar e mensurar a satisfação sexual de indivíduos, com fatores abrangendo tanto os aspectos interpessoais -sentir-se desejado, 
ser capaz de satisfazer o parceiro - como os aspectos físicos individuais- ter vontade de ter sexo, sentir a lubrificação (Carvalheira \& Leal, 2008; Pechorro et al., 2009). Por outro lado, não parece haver uma preocupação, na elaboração de tais instrumentos, com diferenças de satisfação sexual baseadas na orientação sexual dos sujeitos participantes, sendo a maior parte deles referente a vivências heterossexuais. Estudos tem deixado, portanto, esta questão em aberto. Por outro lado, um instrumento que tem sido utilizado em estudos brasileiros é o de Cardoso (2009), que compõe o Questionário de Identidade Corporal, instrumento de auto-relato em que o sujeito responde diretamente a perguntas sobre o quão satisfeito está com diferentes aspectos da sua vida sexual. Este instrumento foi selecionado para os propósitos desta pesquisa, entendendo que o mesmo se baseia no julgamento que o próprio sujeito faz sobre sua vida sexual, independentemente de sua orientação. Resta, portanto, dar atenção a quais os aspectos da vivência homossexual que podem interferir nesta satisfação.

Inicialmente, é preciso se ater ao fato de que existe um número escasso de literatura produzida acerca da satisfação sexual em indivíduos LGB. Uma quantidade considerável das pesquisas que evidentemente tem como objeto de investigação os relacionamentos não-heterossexuais têm como foco aspectos estereotipicamente negativos destes relacionamentos, como o status de HIV (Darbes, Chakravarty, Beougher, Neilands, \& Hoff, 2012) e falta de cometimento monogâmico (Mark, Rosenkrantz, \& Kerner, 2014; Rostosky, Wilcox, Wright \& Randall, 2004), negligenciando variáveis tais como a satisfação sexual, satisfação conjugal, entre outros.

Sobre as diferenças existentes entre níveis de satisfação sexual baseados na orientação sexual dos pares, é possível observar estudos que exploram alguns aspectos divergentes entre estes casais. Em um estudo comparativo feito por Amos y McCabe (2015), com sujeitos heterossexuais, bissexuais e homossexuais, representando um total de 2893 sujeitos, buscava-se investigar a importância da variável "sentir-se sexualmente atraente" para a satisfação sexual, além de outros aspectos da sexualidade. Observou-se que os sujeitos heterossexuais relataram um nível significativamente maior de satisfação sexual e frequência de atividade sexual do que os sujeitos bissexuais, gays e lésbicas. Por outro lado, o estudo feito por Garcia, Lloyd, Wallen, y Fisher (2014) revelou que mulheres lésbicas tem uma maior quantidade de orgasmos previsíveis do que mulheres hetero e bissexuais. 
Após se constatar a existência destas diferenças, é preciso que se questione de onde elas partem. Primeiramente, diversos autores argumentam que a homofobia e estigmatização -que não são vividas pelo casal heterossexualinterferem tanto no bem-estar, como na satisfação do casal gay e lésbico (Belous \& Wampler, 2016; Cerqueria-Santos, Silva, Rodríguez \& Santos, 2016; Frost, 2011; Mark, et al., 2015; Otis, Rostosky, Riggle \& Hamrin, 2006). Por homofobia, entende-se o descrédito, opressão e a violência direcionada aos indivíduos homossexuais ou àqueles presumidos como tal, tendo, portanto, um caráter explícito e observável (Borrillo, 2000; Chávez, Zapata, Petrzelová \& Villanueva, 2018; Eribon \& Haboury, 2003).

Uma outra variável, pertinente a sujeitos LGB e que não está presente em sujeitos heterossexuais, é o nível de abertura -traduzido do inglês, outnessque se refere à o quão público o sujeito é sobre sua orientação sexual nos espaços que frequenta (O'Connor, 2016). O alto nível de abertura tem sido associado na literatura também à níveis maiores de bem-estar individual e níveis menores de outros problemas psicossociais tais como ansiedade e depressão (Kosciw, Palmer \& Kull, 2014), podendo assim, possivelmente, interferir na esfera de satisfação sexual individual.

Além disso, quando se estuda a sexualidade de indivíduos, encontra-se a religião como um fator de forte influência na forma como as pessoas vivem e experimentam sua sexualidade, afetando não somente a idade de iniciação como a frequência de atividade sexual (Cerqueria-Santos, Koller \& Wilcox, 2008; Coutinho \& Miranda-Ribeiro, 2014; Souza, 2013). Ao mesmo tempo, uma posição conservadora generalizada, alimentada pela religiosidade e pelo conservadorismo ético apresentou no estudo de Costa, Peroni, Camargo Pasley e Nardi (2015) associação com o preconceito contra a diversidade sexual. Além destes, outros autores trazem também que existem relações entre religiosidade, sexualidade e nível de preconceito (Costa et al., 2015; Natividade \& De Oliveira, 2009), e que a religiosidade, pautada em ideais conservadores, possui relação estreita com a homofobia explícita (Natividade, 2006; Pereira, Torres, Pereira \& Falcão 2011; Silva, Santos, Licciardi \& Paiva, 2008; Sung, 2014), bem como com homofobia internalizada (CerqueiraSantos, Carvalho, Nunes \& Silveira, 2017).

A homofobia internalizada é um conceito que foi trabalhado inicialmente por Eribon (1999) para uma atitude negativa por parte de indivíduos LGB acerca de sua orientação sexual -nota-se então que está se diferencia da Homofobia explícita já que esta parte principalmente de indivíduos não-homossexuais para com indivíduos LGB. A homofobia internalizada se refere, portanto, à 
rejeição, por parte do próprio indivíduo LGB, à sua identidade homossexual, a outros homossexuais e a tudo o que disser respeito à não-heterossexualidade. Ela parte justamente do processo de internalização da vivência de homofobia a qual este sujeito foi exposto no seu processo de socialização, e serve assim como uma forma de proteção e blindagem contra o conflito gerado pelo seu próprio sistema de crenças (Pereira \& Leal, 2005; Rodrigues, 2010; Souza \& Pereira, 2013).

Diversos fatores da sexualidade de indivíduos LGB tem sido associados à presença Homofobia internalizada. Em uma revisão sistemática da literatura, Lira e Morais (2016) encontraram que, em pesquisas com mulheres lésbicas e bissexuais assim como com homens gays, a homofobia internalizada foi citada como um preditor negativo de Satisfação Sexual. Ao mesmo tempo, estudos com esta população tem reforçado a ideia de que estigma e falta de suporte social colaboram fortemente para uma menor satisfação com o relacionamento em casais do mesmo gênero (Frost, 2011; Otis et al., 2006).

Fica constatado, portanto que existe uma série de fatores que influenciam na vivência e na sexualidade de sujeitos homossexuais e que não necessariamente se apresentam da mesma forma para os heterossexuais. Podendo assim gerar diferenças nos níveis de satisfação destes grupos de sujeitos. Nesta perspectiva, se atentando à demanda na literatura por pesquisas que abordem a parte sexual positiva dos relacionamentos entre pessoas do mesmo sexo, este estudo se propõe a observar a influência das variáveis homofobia internalizada e religiosidade na satisfação sexual de sujeitos em relacionamentos com pessoas do mesmo sexo. A hipótese é de que altos níveis de homofobia internalizada e de religiosidade influenciam de forma negativa a satisfação sexual destes indivíduos.

\section{MÉTODO}

\subsection{Diseño}

Este estudo tem como objetivo observar a influência das variáveis Homofobia Internalizada e Religiosidade na Satisfação Sexual de indivíduos em relacionamentos homossexuais. Para a análise dos dados, as variáveis

\subsection{Participantes}

Participaram do estudo 94 pessoas ( $52,7 \%$ homens, $47,3 \%$ mulheres). Os critérios de inclusão considerados foram: 1) autodeclarar-se homossexual (gay, lésbica ou bissexual); 2) estar vivenciando uma relação conjugal (ou 
união estável) há, pelo menos, um ano ou 3) coabitar com o/a cônjuge há, pelo menos, seis meses; 4 ) ter idade mínima de 18 anos.

\subsection{Instrumentos}

\subsubsection{Questionário sociodemográfico}

Elaborado pelos autores e composto por 24 questões específicas para os objetivos deste estudo, este questionário buscou caracterizar sociodemograficamente os sujeitos (idade, sexo, nível socioeconômico, coabitação, nível de abertura sobre sua orientação sexual, entre outros). Foi realizado um piloto a partir do qual o questionário foi aprimorado.

\subsubsection{Escala de Homofobia Internalizada (Pereira \& Leal, 2005)}

Esta escala avalia os níveis de internalização do preconceito contra homossexuais. Consiste num questionário de 26 itens distribuídos em duas dimensões- percepção interna do estigma e percepção externa do estigma. Todos os itens são redigidos na afirmativa e medidos numa escala de Likert de 5 pontos, desde 1 -discordo totalmente a 5 - concordo totalmente. Seguem alguns itens da escala: 1) homens gays obviamente efeminados fazem-me sentir desconfortável (dimensão 1); 2) prefiro ter parceiros/as sexuais anônimos (dimensão 1); 3) A vida seria mais fácil se eu fosse heterossexual (dimensão 2), dentre outros. O Alpha de Cronbach para o escore geral foi de 0,74 (sendo 0,816 para a primeira dimensão e 0,645 para a segunda).

\subsubsection{Escala de Religiosidade/espiritualidade (Cerqueira-Santos, Koller, \&}

Wilcox, 2008)

Esta escala mede a religiosidade/espiritualidade através dos seguintes aspectos destacados na literatura: afiliações religiosas, prática e crença. 0 instrumento é composto por nove itens e deve ser respondido numa Escala Likert, com valores variando de 1 -Nunca a 5-Sempre. Exemplos de itens da escala: "a religião/espiritualidade tem sido importante na minha vida", "costumo frequentar encontros, cultos ou rituais religiosos", "peço ajuda a Deus para resolver meus problemas", dentre outros. A análise fatorial destes itens confirmou o uso de um único fator $(\mathrm{KMO}=0,86)$, com uma variância explicada de 57\%. A versão original da escala, composta de sete itens, apresentou um alpha Cronbach de 0,87 . $O$ alpha de Cronbach para a amostra deste estudo foi de 0,83 , considerando os itens em forma unifatorial. A escala permite aplicação para indivíduos com ou sem religião, pois explora aspectos da espiritualidade e considera escores mínimos para grupos sem crença alguma. 
Sexualidade: Os sujeitos responderam a sete questões sobre o comportamento sexual. Exemplos das questões são os seguintes: "idade da primeira relação/interação sexual", "idade do parceiro/a com quem teve a primeira relação sexual", "se era do mesmo sexo ou sexo oposto", "idade da primeira relação homossexual", dentre outras. Tais dados não apresentam propriedades psicométricas e foram retirados de Cerqueira-Santos, et al. (2017).

\subsubsection{Escala de Satisfação Sexual (Cardoso, 2009).}

Esta escala está presente como uma dentre as escalas do Questionário sobre Identidade Corporal (QID) desenvolvido pelo Laboratório de Gênero, Sexualidade e Corporeidade do CEFID - UDESC. A escala é composta por cinco itens e deve ser respondida num análogo visual do tipo Likert, com valores variando de 0 - Pouco a 6 - Muito. Exemplos de itens da escala: "O quanto me sinto satisfeito (a) com minha vida sexual?", "O quanto a minha frequência de atividade sexual me satisfaz?", "O quanto atingir o orgasmo me satisfaz sexualmente?", dentre outros. O QIC é um instrumento de caráter subjetivo, portanto, baseado na mensuração do auto-relato.

\subsection{Procedimentos de Coleta de Dados}

Este estudo fez parte de um estudo maior com vistas a investigar diversos aspetos da conjugalidade entre pessoas do mesmo sexo. Para sua realização, os integrantes do grupo de pesquisa entraram em contato com informantes-chave que se enquadravam nos pré-requisitos do estudo para que fizessem parte da amostra e, em seguida, foi utilizado o método snowball (bola de neve) a partir destas participações iniciais para identificar outros potenciais respondentes. Ao todo participaram do estudo 94 indivíduos.

Aos participantes foram explicados os objetivos da pesquisa, esclarecidas algumas questões éticas e solicitada a assinatura do Termo de Consentimento Livre e Esclarecido (TCLE). Somente após a anuência é que a coleta de dados iniciava. Todos os instrumentos foram autoaplicáveis e o tempo médio de aplicação foi de 30 minutos. Durante a aplicação, o pesquisador esteve disponível para retirar quaisquer dúvidas dos sujeitos.

\subsection{Análise de Dados}

Os dados de cada sujeito foram inseridos no Statistical Package for Social Science - SPSS (versão 21) e submetidos a análises descritivas e inferenciais. Realizou-se o cálculo de estatísticas descritivas (frequência, porcentagem e 
média) para a amostra total, com foco nas variáveis utilizadas neste estudo: aspectos sociodemográficos, homofobia internalizada, homofobia externa sofrida, satisfação sexual e índice de religiosidade. Foram feitas comparações de média intergrupos com análise unidirecional ANOVA dentro das variáveis estudadas. Em seguida foi realizado o teste de correlações de Pearson entre as variáveis do estudo e, por fim, uma análise de regressão linear tendo como desfecho a variável satisfação sexual. Homofobia Internalizada, Homofobia Externa, Abertura e Religiosidade foram categorizadas e subdivididas em três níveis cada: Alta, Média ou Baixa. A divisão foi feita partindo-se dos tercis de cada variável, tentando evitar a classificação de indivíduos com escores muitos semelhantes (grupo próximo da média) como polarizados em grupos extremos (somente alta e baixa).

\subsection{Procedimentos Éticos}

O projeto desta pesquisa foi submetido e aprovado pelo Comitê de Ética em Pesquisas envolvendo Seres Humanos da xxx (protocolo número 50367715.0.0000.5546). A pesquisa atende às recomendações bioéticas para pesquisas com seres humanos no que diz respeito à Resolução 466/2012 do Conselho Nacional de Saúde (CNS). Os sujeitos foram informados sobre os princípios bioéticos, também sobre os objetivos e procedimentos do estudo quando convidados para participar voluntariamente da pesquisa e assinaram o Termo de Consentimento Livre e Esclarecido - TCLE.

\section{RESULTADOS}

Assim como consta na Tabela 1, a amostra foi composta por 49 homens e 45 mulheres, o nível de escolaridade dominante foi a Graduação $(65,5 \%)$, seguido pela pós-graduação (18,3\%) e Ensino Médio Completo (12,9\%). No que diz respeito à renda individual, a média foi de $\mathrm{R} \$ 2,094$. Declararam seguir alguma religião $61,7 \%$, sendo a católica a denominação predominante (51,7\%), seguida da espírita (30,7\%), evangélica $(17,6 \%)$ e 6,4\% não responderam qual religião seguem. A amostra apresentou predominantemente um nível de abertura alto $(65,9 \%)$. A média de idade da amostra foi de 28,32 anos, o tempo de relacionamento médio foi de 49,57 meses e, entre os sujeitos que moram juntos, a média de residência conjunta foi de 42,64 meses. A idade média de iniciação sexual foi de 16,55 anos, com a idade média do parceiro de 20,47 anos, enquanto que a idade média da primeira relação sexual homossexual foi de 17,31 anos. 0 sexo do (a) parceiro (a) da primeira relação foi predominantemente do mesmo sexo $(71,7 \%)$, sendo que $46,7 \%$ 
tiveram a primeira relação com namorado (a) e a maior parte da amostra já teve relações sexuais com pessoas de ambos os sexos $(65,2 \%)$.

Tabela 1.

Dados Sociodemográficos $(\mathrm{N}=94)$

\begin{tabular}{|c|c|c|c|c|c|c|}
\hline VARIÁVEIS & & VALORES & & & & \\
\hline & & MIN & MAX & & MÉDIA & DP \\
\hline Idade (em anos) & & 19 & 57 & & 28,32 & 7,71 \\
\hline $\begin{array}{l}\text { Tempo de Relacionamento (em } \\
\text { meses) }\end{array}$ & & 12 & 213 & & 49,57 & 36,43 \\
\hline $\begin{array}{c}\text { Tempo que mora junto (em } \\
\text { meses) }\end{array}$ & & 3 & 164 & & 42,64 & 35,75 \\
\hline Renda Individual (em R\$) & & 0 & 11,000 & & 2,094 & 1954,00 \\
\hline Idade da 1a relação sexual & & 11 & 26 & & 16,55 & 2,57 \\
\hline $\begin{array}{l}\text { Idade do(a) parceiro(a) da 1ạ } \\
\text { relação sexual }\end{array}$ & & 11 & 38 & & 20,47 & 6,10 \\
\hline \multirow[t]{2}{*}{ Idade da 1a relação homossexual } & & 12 & 26 & & 17,31 & 3,04 \\
\hline & & PORCENTAGEM & & FREQUÊNCIA & & \\
\hline \multirow[t]{2}{*}{ Sexo } & Homens & 52,7 & & 49 & & \\
\hline & Mulheres & 47,3 & & 45 & & \\
\hline \multirow[t]{5}{*}{ Escolaridade } & $\begin{array}{l}\text { Ensino Fundamental } \\
\text { Completo }\end{array}$ & 2,2 & & 2 & & \\
\hline & $\begin{array}{l}\text { Ensino Médio } \\
\text { Incompleto }\end{array}$ & 1,1 & & 1 & & \\
\hline & $\begin{array}{l}\text { Ensino Médio } \\
\text { Completo }\end{array}$ & 12,9 & & 12 & & \\
\hline & Graduação & 65,5 & & 61 & & \\
\hline & Pós-graduação & 18,3 & & 17 & & \\
\hline \multirow[t]{3}{*}{ Nível de abertura } & Alta & 65,9 & & 61 & & \\
\hline & Média & 25,2 & & 24 & & \\
\hline & Baixa & 8,9 & & 9 & & \\
\hline Possui religião & & 61,7 & & 58 & & \\
\hline \multirow[t]{3}{*}{ Religião Professada } & Católica & 51,7 & & 34 & & \\
\hline & Evangélica & 17,6 & & 3 & & \\
\hline & Espírita & 30,7 & & 15 & & \\
\hline Mora junto & & 59,8 & & 55 & & \\
\hline $\begin{array}{c}\text { Possuí declaração de União } \\
\text { Estável }\end{array}$ & & 12,8 & & 12 & & \\
\hline $\begin{array}{l}\text { Sexo do(a) parceiro(a) da 1a } \\
\text { relação sexual }\end{array}$ & Mesmo sexo & 71,7 & & 66 & & \\
\hline \multirow{3}{*}{$\begin{array}{c}\text { Sexo das pessoas com quem já } \\
\text { transou }\end{array}$} & Sexo Oposto & 28,3 & & 26 & & \\
\hline & Mesmo sexo & 34,8 & & 32 & & \\
\hline & Ambos os sexos & 65,2 & & 60 & & \\
\hline
\end{tabular}

Uma ANOVA foi utilizada para examinar diferenças na média da Satisfação

Sexual entre subgrupos das variáveis de contraste: "Homofobia Internalizada", "Homofobia Externa" e "Religiosidade". Foram observadas diferenças signi- 
ficativas na Satisfação Sexual entre grupos com "Alta" e "Média" homofobia internalizada $(4,96)$ quando comparados aos grupos com "Baixa" homofobia internalizada $(5,44)$, bem como diferenças entre grupos com níveis médios de religiosidade $(5,40)$ quando comparados a grupos com baixa religiosidade $(4,96)$. Nessa relação, grupos com níveis médios de religiosidade apresentaram maior média de satisfação sexual do que grupos com baixa religiosidade. Além disso, observou-se uma diferença significativa entre sujeitos com alta $(5,02)$ e média $(5,40)$ religiosidade, em que os sujeitos mais religiosos apresentaram uma satisfação sexual menor que os medianos. Por fim, foram observadas diferenças não-significativas entre os grupos com diferentes níveis de homofobia externa sofrida e entre os grupos com "Alta" $(5,02)$ e "Baixa" $(4,96)$ religiosidade. Não foi observada diferença entre os grupos de "Alta" e "Média" homofobia internalizada, já que estes apresentaram a mesma média de satisfação sexual $(4,96)$. Ver Tabela 2.

Tabela 2.

ANOVA para observação de diferenças intra-grupos na satisfação sexual

\begin{tabular}{|c|c|c|c|c|c|c|c|c|}
\hline VARIÁVEIS & ESCORE & $\begin{array}{l}\text { SATISFAÇÃO } \\
\text { SEXUAL }\end{array}$ & $\mathbf{F}$ & $\mathbf{P}$ & MIX & DIF M & $\mathrm{I}, \mathrm{C}$ & SIG \\
\hline \multirow[t]{3}{*}{$\begin{array}{l}\text { Homofobia } \\
\text { Internalizada }\end{array}$} & Alta & 4,96 & 6,31 & 0,003 & Alta/Média & 0,0005 & $\begin{array}{c}-0,398 \\
0,399\end{array}$ & 1,000 \\
\hline & Média & 4,96 & & & Média/Baixa & 0,4832 & $\begin{array}{l}-0,886 \\
-0,079\end{array}$ & 0,015 \\
\hline & Baixa & 5,44 & & & Alta / Baixa & 0,4827 & $\begin{array}{l}-0,843 \\
-0,122\end{array}$ & 0,005 \\
\hline \multirow[t]{3}{*}{ Homofobia Externa } & Alta & 5,18 & 1,98 & 0,114 & Alta / Média & 0,0483 & $\begin{array}{l}-0,423 \\
0,327\end{array}$ & 0,494 \\
\hline & Média & 5,23 & & & Média/Baixa & 0,3397 & $\begin{array}{c}-0,079 \\
0,758\end{array}$ & 0,135 \\
\hline & Baixa & 4,89 & & & Alta / Baixa & 0,2914 & $\begin{array}{l}-0,150 \\
0,733\end{array}$ & 0,263 \\
\hline \multirow[t]{3}{*}{ Religiosidade } & Alta & 5,02 & 4,37 & 0,15 & Alta / Média & 0,3786 & $\begin{array}{l}-0,767 \\
0,009\end{array}$ & 0,058 \\
\hline & Média & 5,40 & & & Média/Baixa & 0,4395 & $\begin{array}{l}0,054 \\
0,824\end{array}$ & 0,021 \\
\hline & Baixa & 4,96 & & & Alta / Baixa & 0,0609 & $\begin{array}{c}-0,333 \\
0,455\end{array}$ & 0,455 \\
\hline
\end{tabular}

Conforme demonstrado na Tabela 3, numa análise de correlação de Pearson das variáveis de interesse com o escore total de Satisfação Sexual, foram 
encontradas correlações significativas positivas com as variáveis "Abertura" $(r=0,232)$ e "Tempo de relacionamento" $(r=0,203)$, além de uma correlação significativa negativa com a variável "Homofobia Internalizada" ( $r=-0,354)$. As correlações entre satisfação sexual e "Religiosidade" e "Homofobia Externa" não foram significativas. Adicionalmente podem ser observadas na tabela correlações significativas positivas entre a variável "Homofobia Internalizada" e as variáveis Religiosidade $(r=0,269)$ e Homofobia Externa $(r=0,326)$ e negativa com a variável Abertura $(r=-0,439)$. Por fim, as variáveis "Abertura" e "Tempo de relacionamento" apresentaram correlação significativa positiva $(r=0,257)$.

Tabela 3.

Correlação de Pearson entre Escalas utilizadas e Dados Demográficos

\begin{tabular}{|c|c|c|c|c|c|c|}
\hline & & RELIGIOSIDADE & $\begin{array}{l}\text { HOMOFOBIA } \\
\text { INTERNALIZADA }\end{array}$ & $\begin{array}{l}\text { HOMOFOBIA } \\
\text { EXTERNA }\end{array}$ & ABERTURA & $\begin{array}{c}\text { TEMPO DE } \\
\text { RELACIONAMENTO }\end{array}$ \\
\hline \multirow[t]{3}{*}{ Satisfação Sexual } & r & 0,027 & $-0,354$ & $-0,003$ & 0,232 & 0,203 \\
\hline & $P$ & 0,801 & 0,001 & 0,978 & 0,028 & 0,054 \\
\hline & N & 91 & 91 & 91 & 90 & 91 \\
\hline \multirow[t]{3}{*}{ Religiosidade } & r & & 0,269 & 0,010 & $-0,227$ & $-0,018$ \\
\hline & $P$ & & 0,009 & 0,923 & 0,28 & 0,863 \\
\hline & N & & 94 & 94 & 93 & 94 \\
\hline \multirow[t]{3}{*}{$\begin{array}{l}\text { Homofobia } \\
\text { Internalizada }\end{array}$} & r & & & 0,326 & $-0,439$ & $-0,165$ \\
\hline & $P$ & & & 0,001 & 0,000 & 0,112 \\
\hline & N & & & 94 & 93 & 94 \\
\hline \multirow[t]{3}{*}{ Homofobia Externa } & r & & & & $-0,085$ & 0,029 \\
\hline & $P$ & & & & 0,419 & 0,779 \\
\hline & N & & & & 93 & 94 \\
\hline \multirow[t]{3}{*}{ Abertura } & r & & & & & 0,257 \\
\hline & $P$ & & & & & 0,013 \\
\hline & N & & & & & 94 \\
\hline
\end{tabular}

A partir dos resultados das variáveis utilizadas na correlação de Pearson, foi elaborado um modelo de regressão linear, tendo como variável dependente a satisfação sexual. Como mostra a Tabela 4, o modelo contou com as variáveis "Homofobia Internalizada", "Homofobia Externa", "Religiosidade", "Tempo de relacionamento" e "Abertura". Embora as variáveis "Religiosidade" e "Homofobia Externa" não tenham apresentado correlação significativa com a satisfação sexual, foi feita a opção por mantê-las tendo em visa sua conexão com a "Homofobia Internalizada" e a força desta variável na correlação com a Satisfação Sexual. À exceção das variáveis "Tempo de relacionamento" e "Abertura", essas variáveis foram estatisticamente significativas como associadas à Satisfação Sexual, sendo que dentre elas, apenas a 
variável "Homofobia Internalizada" interferiu negativamente na satisfação. Tal modelo teve uma variância explicada de $45 \%$ (R de 0,159).

Tabela 4.

Regressão linear para Satisfação Sexual

\begin{tabular}{ccccc}
\hline $\begin{array}{c}\text { MODELO: SATISFAÇÃO } \\
\text { SEXUAL }\end{array}$ & CARGA & ERRO PADRÃO & BETA & P \\
\hline Escore de Religiosidade & 0,161 & 0,085 & 0,199 & 0,063 \\
Homofobia Internalizada & $-0,230$ & 0,082 & $-0,305$ & 0,006 \\
Homofobia Externa & 0,192 & 0,090 & 0,217 & 0,037 \\
Tempo de Relacionamento & 0,201 & 0,137 & 0,153 & 0,144 \\
Abertura & 0,154 & 0,121 & 0,142 & 0,207 \\
\hline
\end{tabular}

\section{DISCUSSÃO}

A produção acadêmica é incipiente no que se refere a estudos sobre a satisfação sexual em casais do mesmo sexo. Os dados obtidos nesta pesquisa apontam que os maiores níveis de satisfação sexual estiveram presentes em sujeitos que apresentaram níveis inferiores de homofobia internalizada e em sujeitos com níveis muito altos ou muito baixos de religiosidade, corroborando assim a hipótese prevista, com o adendo de que níveis muito baixos de religiosidade podem também interferir negativamente na satisfação sexual.

Quanto à interferência da Homofobia Internalizada, este achado está de acordo com o que foi encontrado por Lira e Morais (2016) em sua revisão sistemática da literatura acerca das influências da homofobia internalizada em diferentes aspectos da vida do indivíduo homossexual. Entre os estudos abordados estava o de Henderson, Lehavot e Simoni (2009) que aponta a homofobia internalizada como preditor negativo de satisfação sexual em mulheres lésbicas e bissexuais. No mesmo ano, Frost e Meyer (2009) encontraram uma relação negativa entre estas variáveis em homens gays.

Outra variável inversamente associada à homofobia internalizada foi o nível de abertura. Este dado corrobora o que já foi evidenciado no estudo americano de Kosciw et al., (2014) e no estudo com população do sudoeste chinês de Xu, Zheng, Xu e Zheng (2017) que revelam a importância de se levar em conta como o retraimento na abertura sobre sua orientação sexual é fator provocativo de estresse em minorias sexuais. O'Connor (2016) ressalta ainda que a variável abertura sendo apresentada como um fator redutor de estresse é algo recente, diferente do que era encontrado há 20 anos atrás, quando ter sua orientação sexual exposta previa altos níveis de estresse e adoecimento psicossocial. Este dado pode apontar para prováveis mudanças 
na forma como o meio social e comunidade tem se relacionado com o sujeito homossexual e com sua identidade (Kosciw et al., 2014).

A variável Abertura também apresentou correlação positiva com as variáveis Tempo de relacionamento e Satisfação Sexual. Levando ao entendimento de que, entre os participantes deste estudo, aqueles casais que se encontram juntos a mais tempo são mais abertos sobre sua sexualidade nos ambientes de frequentam, assim como possuem um maior nível de Satisfação Sexual. Sabe-se que a publicização da orientação sexual, na juventude, parece seguir uma linha que vai do menor para o maior risco social, onde os primeiros a saberem são outros sujeitos LGB, seguidos de amigos próximos, que podem ou não ser homossexuais, colegas de trabalho e por fim membros da família, assim como que este processo leva certo tempo, devido ao nível de exposição a fatores estressores aos quais o sujeito homossexual é colocado ( $\mathrm{O}^{\prime} \mathrm{Connor}$, 2016)

A variável religiosidade pareceu exercer sua influência nos polos da amostra. Sujeitos homossexuais muito ou pouco religiosos tiveram menores níveis de Satisfação Sexual do que os sujeitos com níveis médios. A literatura traz que a religiosidade diz sobre o comportamento sexual dos sujeitos, tanto em aspectos como iniciação da atividade sexual, busca de prazer sexual, como na própria satisfação sexual (Byers, O’Sullivan \& Brotto, 2016; Cerqueria-Santos et al., 2008; Coutinho \& Miranda-Ribeiro, 2014; Souza, 2013).

Essa característica reguladora da religiosidade às práticas sexuais, quando aplicada ao sujeito homossexual, pode causar um aumento em sua homofobia internalizada, ou seja, em um aumento de percepção negativa sobre si mesmo e sobre sua identidade homossexual (Cerqueira-Santos et al., 2017). Portanto, levando em conta o que já foi discutido sobre como a homofobia internalizada provocou um declínio na satisfação sexual dos sujeitos participantes deste estudo, é possível inferir que a internalização da homofobia pode ter atuado como efeito mediador que corrobora em uma baixa satisfação sexual.

Ao mesmo tempo, a religiosidade baixa pode pressupor um afastamento por parte do sujeito homossexual das religiões em si, possivelmente pela carga dogmática e de ideais punitivos à pratica homossexual muitas vezes cultivados, mesmo que em níveis distintos, nestas religiões (Costa et al., 2015; Natividade \& De Oliveira, 2009). Não é de se estranhar, portanto, 
que a religiosidade tenha se mostrado como um fator influente também no presente estudo.

Ainda sob uma outra perspectiva, Souza (2013) encontrou a religião como um dentre os fatores que podem ser determinantes de satisfação sexual. Foi observado que entre os sujeitos deste estudo, 61,7\% afirmaram possuir religião, entretanto, não seria viável explorar diferenças inter-grupos religiosos, pois a grande maioria se declarou católica $(51,7 \%)$, enquanto que os que restaram se dividiram de forma bastante irregular dentro dos outros grupos religiosos.

Este viés católico na amostra pode ser apontado como uma dentre as limitações encontradas nesta pesquisa. Além disso, o fato da amostra ter sido composta por indivíduos de maioria jovem e com pouco tempo de relacionamento, pode ter gerado um viés no resultado encontrado. Schmiedeberg e Schroder (2015) apontam que, em relacionamentos heterossexuais, a satisfação sexual sofre influência negativa do tempo de duração do relacionamento, de forma que com o passar dos anos esta tende a diminuir. Os dados obtidos na presente amostra, relevaram que o tempo de duração do relacionamento exerceu influência positiva na satisfação sexual. Isso pode ter ocorrido pois os casais do estudo apresentaram uma média de tempo de relacionamento relativamente baixa (49,77 meses), quando comparada ao estudo de Schmiedeberg e Jette Schroder onde média de duração era maior. Ainda se tratando do ponto de vista metodológico, o fato de os sujeitos participantes integrarem outro estudo maior sobre conjugalidade pode ter gerado respostas semelhantes entre os pares de convivência.

Neste estudo, encontramos a presença da variável homofobia internalizada como um forte preditor negativo de satisfação sexual, ao mesmo tempo, observamos a relação desta variável com outras tais como a religiosidade, homofobia externa sofrida, nível de abertura e tempo de relacionamento. Como já foi discutido, existe suporte literário para cada uma destas relações. O que reforça a importância de se observar esta variável que está presente e exerce sua influência na saúde psicológica, social e afetiva do indivíduo LGB.

Do ponto de vista conceptual, uma exploração na literatura atual sobre o tema da satisfação sexual voltada para casais heterossexuais traz que este construto se correlaciona de forma positiva com outras variáveis, tais como: ter atitudes positivas sobre sexo (Dosch, Belayachi \& Van der Linden, 2015), e com frequência de atividade sexual (Frederick, Brian, Gillespie \& García, 2016; Mcnulty, 
Wenner \& Fisher, 2014; Schoenfeld, Loving, Pope, Houston \& Stulhofer, 2016). No entanto, em pesquisa nas principais bases de dados de periódicos científicos do Brasil (SCIELO e PEPSIC) não foi possível encontrar estes temas investigados com foco em casais do mesmo sexo.

A baixa produção científica acarreta em prejuízo na forma de se conhecer e trabalhar os diferentes tipos de vivências da sexualidade entre os sujeitos de diferentes orientações. O que invisibiliza demandas que são específicas dos grupos de minorias sexuais. Pensando sob esta perspectiva, produzir academicamente sobre satisfação sexual e outros aspectos da sexualidade LGB é uma forma de respeitar e reconhecer este construto em sua totalidade e pode servir de base para que psicólogos e outros profissionais da saúde compreendam e desenvolvam suas práticas sob uma orientação mais sólida e menos intuitiva.

Agradecimentos: Agradeço aos participantes da pesquisa, bem como ao Prof. Phd. Elder Cerqueira Santos pela orientações.

Financiamento: Trabalho monográfico para conclusão do curso de graduação em psicologia pela Universidade Federal de Sergipe.

\section{REFERÊNCIAS}

Amos, N., \& McCabe, M. (2015). The importance of feeling sexually attractive: Can it predict an individual's experience of their sexuality and sexual relationships across gender and sexual orientation? Interational Journal of Psychology, 52(5), 349-424. https://doi.org/10.1002/ijop.12225

Apt, C., Hurlbert, D.F., Pierce, A.P., \& White, C.L. (1996). Relationship satisfaction, sexual characteristics and the psychological well-being of women. Canadian Journal of Human Sexuality, 5(3), 195-210. Recuperado em: http://psycnet.apa. org/record/1997-02985-005

Ashdown, B. K., Hackathorn, J., \& Clark, E. M. (2011). In and out of the bedroom: Sexual satisfaction in the marital relationship. Journal of Integrated Social Sciences, 2(1), 40-57. Recuperado em: http://www.jiss.org/archive/2-1.php

Belous, C. K., \& Wampler, R. S. (2016). Development OD he gay and lesbian relationship satisfaction scale. Journal of marital and Family Therapy, 42(3), 451-465. https:// doi.org/10.1111/jmft.12158

Borrillo, D. (2000). L'Homophobie. Paris: Presses Universitaires de France.

Byers, E. S., O'Sullivan, L. F. \& Brotto, L. A. (2016). Time Out from Sex or Romance: Sexually Experienced Adolescents' Decisions to Purposefully Avoid Sexual Activity or Romantic Relationships, Journal of Youth Adolescence, 45(5), 831-845. https:// doi.org/10.1007/s10964-016-0447-9

Cardoso, J. (2003). Sexualidade masculina pós-lesão vertebro-medular. (Dissertação de Doutoramento não publicada). Instituto de Ciências Biomédicas de Abel Salazar da Universidade do Porto, Porto. Recuperado em: http://hdl.handle. net/10400.12/1621.

Cardoso, F. L., Savall, A. C., Sabbag, S., Mendes, A. K., \& Beltrame, T. S. (2009). Implicações do conhecimento corporal no comportamento sexual. Revista Brasileira 
de Educação Física e Esporte, 23(4), 345-354. http://dx.doi.org/10.1590/S180755092009000400004.

Carvalheira, A., \& Leal, I. (2008). Os determinantes da satisfação sexual feminina: um estudo português. Revista Internacional de Andrologia, 6(1), 3-7. https://doi. org/10.1016/S1698-031X(08)72559-7

Cerqueira-Santos, E., Carvalho, C. A. S. G., Nunes, L. M., \& Silveira, A. P. (2017). Homofobia Internalizada e Religiosidade entre casais do mesmo sexo. Temas em Psicologia, 25(2), 691-702. https://dx.doi.org/10.9788/TP2017.2-15

Cerqueira-Santos, E., Silva, B. B., Rodrigues, H. S., \& Santos, L. (2016) Homofobia internalizada e satisfação conjugal em homens e mulheres homossexuais. Contextos Clínicos, 9(2), 148-158. https://dx.doi.org/10.4013/ctc.2016.92.01

Cerqueria-Santos, E., Koller, S. H., \& Wilcox, B. (2008). Condom Use, Contraceptive Methods, and Religiosity among Youths of Low Socioeconomic Level. The Spanish Journal of Psychology, 11(1), 94-102. https://doi.org/10.1017/ S1138741600004157

Chávez, M., Zapata, J., Petrzelová, J. \& Villanueva, G. (2018). La diversidad sexual y sus representaciones en la juventud. Psicogente, 21(39), 62-74. http://doi. org/10.17081/psico.21.39.2822

Costa, A. B., Peroni, R. O. Camargo, E. S., Pasley, A., \& Nardi, H. C. (2015) Prejudice Toward Gender and Sexual Diversity in a Brazilian Public University: Prevalence, Awareness, and the Effects os Education. Sexuality Research and Social Policy, 12 (4), 261-272. http://doi.org/10.1007/s13178-015-0191-z

Coutinho, R., \& Miranda-Ribeiro, P. (2014). Religião, religiosidade e iniciação sexual na adolescência e juventude: lições de uma revisão bibliográfica sistemática de mais de meio século de pesquisas. Revista Brasileira de Estudos de População, 31(2), 333-365. http://dx.doi.org/10.1590/S0102-30982014000200006

Darbes, L., Chakravarty, D., Neilands, T., Beougher, S. \& Hoff, C. (2012). Sexual risk for HIV among gay male couples: A longitudinal study of the impact of relationship dynamics. Archives of Sexual Behavior, 43(1), 47-60. http://doi.org/10.1007/ s10508-013-0206-x

Davidson, J. K., Darling, C.A., \& Norton, L. (1995). Religiosity and the sexuality of women: Sexual behavior and exual satisfaction revisited. The Journal of Sex Reserach, 32(3), 235-243. http://doi.org/10.1080/00224499509551794

Davis, J., \& Petretic-Jackson, P. (2000). The impact of childhood sexual abuse on adult interpersonal functioning: A review and synthesis of the empirical literature. Agression and Violent Behavior, 5(3), 291-328. https://doi.org/10.1016/S13591789(99)00010-5

Davison, S. L., Bell, R. J., LaChina, M., Holden, S. L., \& Davis, S. R. (2009). The relationship between self-reported sexual satisfaction and general well-being in women. Journal of Sexual Medicine, 6(10), 2690-2697. http://dx.doi.org/10.1 111/j.1743-6109.2009.01406.xMedline:19817981

Delamater, J. (1991). Emotions and sexuality. In K. McKinney \& S. Sprecher (Eds.), Sexuality in close relationships New Jersey: Lawrence Erlbaum Associates.

Dosch, A., Belayachi S., \& Van der Linden, M. (2015). Implicit and Explicit Sexual Attitudes: How Are They Related to Sexual Desire and Sexual Satisfaction in Men and Women? The Journal of Sex Research, O(0), 1-14, https://doi.org/10.1080/0 0224499.2014 .1003361

Eribon, D. (1999). Réflexions sur la question gay. Paris: Fayard.

Eribon, D., \& Haboury, F. (2003). Dictionnaire des cultures gay et lesbiennes. Paris: Editions Larousse. 
Frederick, D. A., Brian, K. L., Gillespie, J., \& Garcia, J. R. (2016): What Keeps Passion Alive? Sexual Satisfaction is associated with Sexual Communication, Mood Setting, Sexual Variety, Oral Sex, Orgasm, and Sex Frequency in a National U.S. Study, The Journal of Sex Research, 00(00), 1-16. https://doi.org/10.1080/00224 499.2015.1137854

Frost, D. M. (2011). Stigma and intimacy in same-sex relationships: A narrative approach. Journal of Family Psychology, 25(1), 1-10. https://doi.org/10.1037/ a0022374

Frost, D. M., \& Meyer, I. H. (2009). Internalized homophobia and relationship quality among lesbians, gay men, and bisexuals. Journal of Counseling Psychology, 56, 97-109. http://dx.doi.org/10.1037/a0012844

Garcia, J. R., Lloyd, E. A., Wallen, K., \& Fisher, H. E. (2014). Variation in orgasm ocurrence by sexual orientation in a sample os U.S. singles. The Journal os Sexual Medicine, 11(11), 2645-2652. https://doi.org/10.1111/jsm.12669

Henderson, A. W., Lehavot, K., \& Simoni, J. M. (2009). Ecological models of sexual satisfaction among lesbian/bisexual and heterosexual women. Archives of Sexual Behavior, 38(1), 50-65. https://doi.org/10.1007/s10508-008-9384-3

Kosciw, J. G., Palmer, N. A., \& Kull, R. M. (2014). Reflecting Resiliency: Openness about sexual orientation and/or gender identity and its relationship to well-being and educational outcomes for LGBT students. American Journal of Communiti Pychology, 55(1-2), 176-178. https://doi.org/10.1007/s10464-014-9642-6

Lira, A. N., \& Morais, N. A. (2016). Famílias constituídas por lésbicas, gays e bissexuais: revisão sistemática de literatura. Temas em Psicologia, 24(3), 1051-1067. https://doi.org/10.9788/TP2016.3-14Pt

Mark, K. P., Garcia, J. R., \& Fisher, H. E. (2015). Percieved emotional and sexual satisfaction acress sexual relationship contexts: Gender and sexual orientation differences and similarities. The Canadian Journal of Human Sexuality, 24(2), 120-130. https://doi.org/10.313B/cjhs.242-A8

Mark, K., Rosenkrantz, D., \& Kerner, I. (2014). "Bi"ing into monogamy: Attitudes toward monogamy in a sample OD bisexual-identified adults. Psychology of Sexual Orientation and Gender Diversity, 1(3), 263. http://dx.doi.org/10.1037/ sgd0000051

McNulty, J. K., Wenner, C. A., \& Fisher, T. D. (2014). Longitudinal Associations among Relationship Satisfaction, Sexual Satisfaction, and Frequency of Sex in Early Marriage. Archives of Sexual Behaviour, 45(1), 85-97. https://doi.org/10.1007/ s10508-014-0444-6

Natividade, M. (2006). Homossexualidade, gênero e cura em perspectivas pastorais evangélicas. Revista brasileira de Ciências Sociais, 21(61), 115-132. http://dx.doi. org/10.1590/S0102-69092006000200006

Natividade, M. T., \& De Oliveira, L. (2009). Sexualidades ameaçadoras: religião e homofobia (s) em discursos evangélicos conservadores. Sexualidad, Salud y Sociedad: Revista Latinoamericana, (2), 121-161. Recuperado em: http:// www.e-publicacoes.uerj.br/index.php/SexualidadSaludySociedad/issue/view/7/ showToc.

O'Connor, J. (2016). Outness, Self-Esteem, Stigma, Psychological Health and Mobile Dating Application Use in Gay and Bissexual Men. Monografia. Dublin Business School, Dublin, Irlanda.

Otis, M. D., Rostosky, S. S., Riggle, E. D. B., \& Hamrin, R. (2006). Stress and relationship quality in same-sex couples. Journal of social and Personal Relationships, 23(1), 81-99. https://doi.org/10.1177/0265407506060179 
Pechorro, P. F. S. (2006). Funcionamento sexual e ciclo de vida em mulheres Portuguesas. (Tese de mestrado). Instituto Superior de Psicologia Aplicada, Lisboa, Portugal. https://doi.org/10.14417/ap.388

Pechorro, P., Diniz, A., Almeida, S., \& Vieira, R. (2009). Validação de uma versão feminina do Índice de Satisfação Sexual. Laboratório de Psicologia, 7(1), 45-56. Recuperado em: http://hdl.handle.net/10400.12/1255.

Pereira, H., \& Leal, I. (2005). Medindo a homofobia internalizada: A validação de um instrumento. Análise Psicológica, 23(3), 315-318. Recuperado em http://www. scielo.mec.pt/pdf/aps/v23n3/v23n3a10.pdf

Pereira, C. R., Torres, A. R. R., Pereira, A. S. P., \& Falcão, L. (2011). Preconceito contra homossexuais e representações sociais da homossexualidade em seminaristas católicos e evangélicos. Psicologia: Teoria e Pesquisa, 27(1), 73-82. http://dx.doi. org/10.1590/S0102-37722011000100010.

Rodrigues, P. (2010). Homofobia Internalizada e Suicidalidade em Jovens LGB e não LBG. Les online, 2(2), 22-34. Recuperado em: http://www.lespt.org/lesonline/ index.php?journal=lo\&pa-ge=article\&op=viewFile\&path\%5B\%5D=35\&pa-th\%5B\%5D=34

Rostosky,S.S., Wilcox, B.L., Wright, M.L.C., \& Randall, B.A. (2004). Theimpact ofreligiosity on adolescent sexual behavior: A review of the evidence. Journal of Adolescent Research, 19(6), 677-697. https://doi.org/10.1177/0743558403260019

Schmiedeberg, C. \& Schroder, J. (2015). Does Sexual Satisfaction Change with Relationship Duration? Archives of Sexual Behaviour, 45(1), 99-107. https://doi. org/10.1007/s10508-015-0587-0

Schoenfeld, E. A.; Loving, T. J.; Pope, M. T.; Huston, T. L. \& Stulhofer, A. (2016). Does Sex Really Matter? Examining the Connections Between Spouses/Nonsexual Behaviors, Sexual Frequency, Sexual Satisfaction, and Marital Satisfaction. Archives of Sexual Behavior, 46(2), 489-501. https://doi.org/10.1007/s10508015-0672-4

Silva, C. G. dá, Santos, O. A., Licciardi, D. C., \& Paiva, V. (2008). Religiosidade, juventude e sexualidade: entre a autonomia e a rigidez. Psicologia em estudo, 13(4), 683-692. http://dx.doi.org/10.1590/S1413-73722008000400006

Souza, E. M., \& Pereira, S. J. N. (2013). (Re) produção do heterossexismo e da heteronormatividade nas relações de trabalho: a discriminação de homossexuais por homossexuais. Revista de Administração Mackenzie, 14(4), 76-105. http://dx.doi. org/10.1590/S1678-69712013000400004

Souza, I. M. N. (2013). Satisfação Sexual e Qualidade de Vida da Mulher no Climatério. (Dissertação de Mestrado). Universidade Fernando Pessoa, Porto. Recuperado em: http://hdl.handle.net/10284/3862

Sung, J. M. (2014). Prosperidade sim, família homossexual, não! A nova classe média evangélica. Psicologia USP, 26(1), 43-51. http://dx.doi.org/10.1590/01036564D20140011

Xu, W., Zheng, L., Xu, Y., \& Zheng, Y. (2017). Internalized homophobia, mental health, sexual behaviors, and outness of gay/bisexual men from Southwest China. International Journal for Equity in Health, 16(1), 16-36. https://doi.org/10.1186/ s12939-017-0530-1 